\title{
LA CONSTRUCCIÓN DEL CONOCIMIENTO SOBRE LA CERÁMICA DE MOMENTOS TEMPRANOS DEL VALLE DE YOCAVIL: UN CAMINO LARGO Y SINUOSO
}

\author{
Romina C. Spano \\ Museo Etnográfico Juan B. Ambrosetti (UBA) - CONICET Arriola 232 (1437) C.A.B.A. \\ rominasp77@yahoo.com.ar
}

Presentado el: 31/08/2009 - Aceptado 23/12/2009

\section{Resumen}

La historia de las investigaciones ceramológicas en el NOA se organizó recientemente en un modelo de cuatro etapas, abarcando desde los inicios del siglo XX al presente (Ramundo 2008). Aquí ponemos a prueba la aplicabilidad de dicha propuesta, abordando el devenir de los estudios sobre la alfarería de las sociedades tempranas del Valle de Yocavil; proponemos que las etapas planteadas para el ámbito del Noroeste, en el caso de Yocavil constituyen instancias cronológicas sucesivas en el desarrollo de la investigación arqueológica y, a la vez, perspectivas sincrónicas respecto a la cerámica como objeto de estudio.

Palabras clave: Estudios ceramológicos - cerámica temprana - Valle de Yocavil

\begin{abstract}
The history about pottery research in Northwestern Argentina has been recently organized in a model of four stages, from the beginning of the twentieth century until the present time (Ramundo 2008). The aim of this paper is to prove the applicability of this model, addressing the evolution of pottery research of early societies at the Yocavil Valley. We assert that the stages proposed for the development of pottery research in the northwest of Argentina, when applied to the case of Yocavil, not only consist of consecutive chronological instances but, going beyond the diachrony, they also represent synchronical ways to address ceramic objects.
\end{abstract}

Key words: Pottery research - early pottery - Yocavil Valley

\section{Introducción}

En el Noroeste Argentino (NOA) la tradición del estudio de la cerámica arqueológica se desarrolló en paralelo con la historia misma de la disciplina. El valle de Santa María o Yocavil (porción sur de los valles Calchaquíes, provincias de Catamarca, Salta y Tucumán) es un caso paradigmático al respecto, ya que el interéspor su pasado se remonta al último cuarto del siglo XIX (Nastri 2004). Décadas de investigación arqueológica resultaron en una construcción del pasado calchaquí asociada fuertemente a la cultura Santa María del período Tardío o de Desarrollos Regionales - 900 al 1480 AD (Tarragó 1999) -. Sin embargo, las sociedades que antecedieron en el tiempo a las poblaciones tardías quedaron relegadas en el relato arqueológico, dando como resultado un cierto "vacío" de información (Scattolin 2000) respecto a los grupos agropastoriles del período Temprano o Formativo ${ }^{1}$ - momento histórico del NOA extendido entre el 600 AC y el 600 AD (Tarragó 1999), y definido localmente en sentido amplio como el lapso comprendido entre el 500 AC y el 1000 AD (Scattolin 2006b) - 
Recientemente se ha propuesto un modelo historiográfico de cuatro etapas sucesivas para organizar el estudio de la investigación ceramológica en el NOA (Ramundo 2008). El objetivo del presente trabajo es evaluar las posibilidades de aplicación de dicho modelo pensado para el NOA en general, a un caso particular, el de la cerámica formativa de Yocavil No se pretende aquí lograr un estudio exhaustivo acerca del estado de conocimiento sobre este universo alfarero, sino examinar si una propuesta tan amplia como la aludida, resulta adecuada para la organización de la trayectoria de la investigación de una problemática acotada.

A nivel metodológico, se realizará una revisión bibliográfica de la investigación ceramológica en el Valle que haya involucrado materiales tempranos. Se avanzó partiendo de la hipótesis de que el modelo historiográfico planteado para los estudios de la alfarería del NOA por Ramundo (2008), se corresponde sincrónicamente con las tendencias en las investigaciones sobre la cerámica temprana en Yocavil.

\section{La investigación ceramológica en el NOA. El valle de Yocavil}

En la construcción del conocimiento del pasado indígena, los objetos cerámicos han jugado un rol protagónico, fundamentalmente las piezas decoradas (Balesta y Williams 2007). El devenir histórico de los estudios de la alfarería arqueológica en el NOA ha sido analizado recientemente por Ramundo (2008); en ese estudio, la autora consideró tanto el contexto sociohistórico que operó de telón de fondo de las investigaciones, como el estatus otorgado a los objetos cerámicos por las mismas, abarcando un lapso extendido desde el año 1900 hasta nuestros días. El modelo propuesto reviste interés en virtud de que, si bien existen estudios que abordan aspectos o etapas de la historia de la arqueología argentina (e. g. Fernández 1982; Madrazo 1985), así como producciones de objetivos más acotados sobre la investigación ceramológica (García y Kusch 1994; Balesta y Williams 2007), no se cuenta con otro estudio historiográfico enfocado hacia los análisis cerámicos desde sus inicios hasta la actualidad, en el área geográfica particular del NOA. La propuesta puede sintetizarse a grandes rasgos en el cuadro que sigue (para un mayor detalle, remitimos a Ramundo 2008):

En el ámbito del NOA, la región calchaquí brinda la posibilidad de realizar un seguimiento de esta serie de etapas, dado que los acercamientos arqueológicos a su pasado se iniciaron en los albores de la disciplina en el siglo XIX, y continúan en la actualidad a través de las tareas de varios equipos de investigación. Haciendo foco específicamente en el valle de Santa María o Yocavil (Figura 1), se cuenta con un nutrido corpus de información acerca del pasado de las poblaciones que lo habitaron después del $900 \mathrm{AD}$, es decir, durante el período Tardío o de Desarrollos Regionales, y posteriormente en momentos de ocupación incaica y contacto Hispano-indígena (e. g. Bengtsson et al. 2001; Nastri 2008; Tarragó y González 2008).

Como efecto acumulativo de más de un siglo de investigaciones, el Valle ha sido asociado en el relato arqueológico del NOA con estas poblaciones tardías, teniendo como ícono material a las grandes urnas funerarias santamarianas. La contraparte de esta situación es un escaso conocimiento de la alfarería anterior al $900 \mathrm{AD}$ y de los procesos sociales de momentos formativos. A continuación realizaremos una breve revisión de la construcción del pasado profundo del valle de Yocavil basada en los estudios de la cerámica de las sociedades aldeanas que lo habitaron, siguiendo un orden cronológico coincidente con las etapas del modelo descripto; el énfasis estará puesto en producciones que aluden a la mitad meridional 


\begin{tabular}{|c|c|c|}
\hline Etapa & Extensión temporal & Características principales \\
\hline $\begin{array}{l}1^{\text {a }} \text { - La primacía del } \\
\text { objeto cerámico }\end{array}$ & $\begin{array}{l}\text { Inicios del siglo XX - } \\
1948\left(^{\star}\right) \\
\\
\left(^{*}\right) \text { la autora incluye } \\
\text { asimismo estudios } \\
\text { de años previos al } \\
1900\end{array}$ & $\begin{array}{l}\text { - Interés por las piezas decoradas, de contextos } \\
\text { funerarios } \\
\text { - Acopio de piezas enteras para conformar grandes } \\
\text { colecciones } \\
\text { - Prácticas de campo: recolección y excavación } \\
\text { selectiva } \\
\text { - Tendencia al descarte de la cerámica ordinaria } \\
\text { - Propuestas de cronologias y tipologías intuitivas } \\
\text { - Clasificación de la variabilidad cerámica en base } \\
\text { a un fundamento geográfico (la antigüedad de las } \\
\text { poblaciones no fue resuelta) }\end{array}$ \\
\hline $\begin{array}{l}2^{\text {a }} \text { - Cerámica para la } \\
\text { cronología o La } \\
\text { cerámica como } \\
\text { documento de } \\
\text { identidad de las } \\
\text { culturas }\end{array}$ & $1948-1980$ & $\begin{array}{l}\text { - Énfasis en la cronología y en la importancia de los } \\
\text { contextos de hallazgo (propuestas teóricas de la } \\
\text { ecología cultural) } \\
\text { - Objeto cerámico: considerado el indicador en la } \\
\text { identificación de culturas (enfoques de corte } \\
\text { histórico-cultural) } \\
\text { - Eje de gran parte de las investigaciones: } \\
\text { determinación de secuencias culturales y de } \\
\text { contextos de las distintas culturas, en base a la } \\
\text { alfarería decorada (e. g. Bennet et al.1948; } \\
\text { González 1955, 1960) } \\
\text { - Necesidad de criterios claros de clasificación de la } \\
\text { cerámica: elaboración del manual de Serrano } \\
\text { (1958) y conformación de la Primera Convención } \\
\text { Nacional de Cerámica (1966) }\end{array}$ \\
\hline $\begin{array}{l}3^{a} \text { - La cerámica como } \\
\text { parte de un todo } \\
\text { complejo }\end{array}$ & $1980-1990$ & $\begin{array}{l}\text {-Cerámica: un elemento más del registro } \\
\text { arqueologico } \\
\text {-Investigación ceramológica: línea de evidencia } \\
\text { acerca de aspectos no jerarquizados hasta ese } \\
\text { momento (procesos de manufactura, fuentes de } \\
\text { materia prima, funcionalidad, etc.) } \\
\text {-Incidencia de la arqueologia procesual e } \\
\text { introducción de estudios etnoarqueológicos, } \\
\text { técnicas arqueométricas y experimentación }\end{array}$ \\
\hline $\begin{array}{l}4^{\text {a }} \text { - El estado actual de } \\
\text { los estudios cerámicos }\end{array}$ & $\begin{array}{l}1990 \text { hasta la } \\
\text { actualidad }\end{array}$ & $\begin{array}{l}\text { - Profusión de planteos vinculados con corrientes } \\
\text { post-procesuales, acerca de los aspectos } \\
\text { ideológicos de las sociedades pasadas } \\
\text { - Introducción de abordajes semióticos y modelos } \\
\text { estructuralistas aplicados al estudio de la } \\
\text { iconografía } \\
\text {-Estudios contextuales de colecciones } \\
\text { - Valorización del estudio de la alfarería doméstica } \\
\text { u ordinaria } \\
\text {-Incursión de exponentes del mundo extra- } \\
\text { académico en los estudios cerámicos (ceramistas y } \\
\text { artistas plásticos) }\end{array}$ \\
\hline
\end{tabular}

del Valle, debido a que esta área ha sido la más visitada por los estudios acerca del Formativo local.

\section{Coleccionismo y saqueo (Primera etapa)}

El coleccionismo signó de alguna manera el devenir de las concepciones respecto a las sociedades del pasado en Yocavil en los años venideros; la apreciación estética occidental guió los criterios de selección respecto a qué objetos cerámicos recolectar y conservar. Las incursiones de Liberani y Hernández (1956 [1877]) en el Valle - las cuales señalan el inicio de las investigaciones arqueológicas en la Argentina -, implicaron un acopio de muchas piezas cerámicas, entre otros objetos; curiosamente, se incluyeron sólo dos piezas de 


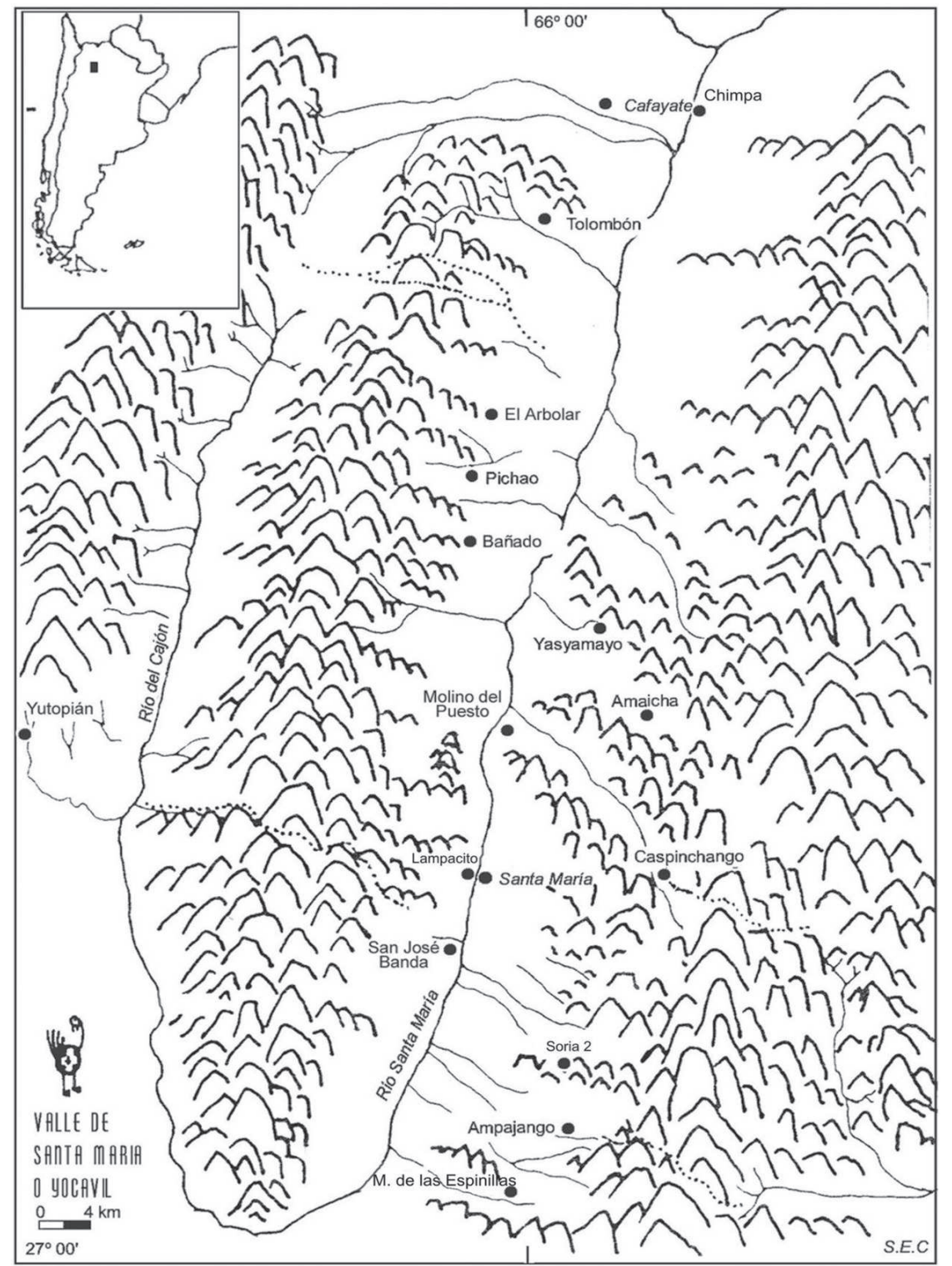

Figura 1. Valle de Yocavil, con la ubicación de algunos sitos con ocupaciones formativas (modificado de Álvarez Larrain et al. 2006).

momentos formativos (Scattolin 2006b). La recuperación de materiales tempranos se inició con Bruch en la primera década del siglo XX; las colecciones Zavaleta del museo Etnográfico de Buenos Aires, y Bravo del museo de Cafayate, poseen materiales de dichas épocas (Tarragó y Scattolin 1999).

Según Scattolin (2000) las intervenciones de los coleccionistas habrían tenido implicancias cruciales en el estado de conocimiento posterior de la cerámica temprana. El saqueo llevado a cabo por M. Zavaleta redundó en la obtención de miles de piezas, cuyos contextos se desconocen debido a las precauciones que aquél tomó para impedir interferencias en el negocio del tráfico de objetos arqueológicos (Tarragó 2003); los destinos de estas piezas se 
reparten entre el museo Etnográfico, el museo de Chicago y el de Berlín. También tuvo incidencia el accionar de R. Schreiter, quien conformó colecciones en base a excavaciones de cementerios prehispánicos del Valle; si bien muchos objetos se encuentran hoy en el museo Etnográfico y en el Instituto de Arqueología de Tucumán (Tarragó 2003), otros están en instituciones extranjeras. Como resultado de estas prácticas, las posibilidades de hallazgos posteriores se habrían visto reducidas críticamente (Scattolin 2000); al mismo tiempo, no se generaron publicaciones de una envergadura acorde respecto a las asociaciones contextuales de las piezas (Tarragó 2003): lo importante eran las piezas en sí.

Diferente fue el caso de las expediciones de V. Weiser, quien plasmó en sus libretas información contextual relevante vinculada con las piezas extraídas que conformarían la colección Muñiz Barreto, hoy depositada en el museo de La Plata. Pero para el caso que nos incumbe, es pertinente señalar que a diferencia del valle de Hualfín, en el cual se excavaron grandes cementerios tempranos, en Yocavil sólo se encontraron dos tumbas de ese momento, con la consiguiente escasez de piezas del Formativo en la Muñiz Barreto (Scattolin 2000).

Por otra parte, exponentes de esta etapa pionera de la investigación ceramológica en el NOA, como Ambrosetti (1896) o Debenedetti (1912), se concentraron en las piezas santamarianas en sus trabajos descriptivos o interpretativos; pero sí existe un trabajo de Ambrosetti (1892) en el cual describió piezas cerámicas donadas al museo de Entre Ríos, atribuyendo a los calchaquíes de la época de la conquista, ceramios que hoy se sabe corresponden a estilos formativos (Nastri 2004). El pasado indígena concebido por los investigadores de ese momento era más bien homogéneo y asociado a las poblaciones calchaquíes del momento de contacto con los incas o con los españoles, hecho que encuentra su contraparte metodológica en la búsqueda de vínculos entre los objetos recuperados y el conocimiento plasmado en las fuentes etnohistóricas y etnográficas. Si bien se tenía una idea de pasado con profundidad temporal, que tomaba en cuenta el factor diacrónico que atravesaba la trayectoria de la población indígena, se carecía de herramientas metodológicas para poder aplicarla a los materiales; existieron referencias a la diacronía de la ocupación indígena e incluso propuestas de periodificación, pero su carácter fue excepcional (Nastri 2004).

\section{Cronología y culturas: mirando hacia el sur (Segunda etapa)}

Desde 1948 la cerámica se erigió como el objeto por excelencia en la construcción de secuencias estilísticas y cronológicas. Esto se manifestó a partir de la publicación de Bennet y colaboradores de ese año, en la cual se incluía al valle de Yocavil en el área central del NOA, junto con los valles Calchaquí y de Lerma, parte de la Puna y de las Selvas Occidentales; a esta área le atribuyó los estilos cerámicos Candelaria y Tafí.

Un trabajo que implicó un quiebre en el modo de entender el pasado en el NOA, fue el de A. R. González (1955), en el cual expresó su preocupación central en años venideros: la necesidad imperiosa de establecer cronologías de las áreas más importantes de la región. Planteó entonces una secuencia cultural relativa para el área central del noroeste, a partir de pruebas estratigráficas, superposiciones y seriación de tumbas, correlaciones tipológicas y referencias a contribuciones de otros arqueólogos. La cerámica fue un elemento de suma importancia en este análisis, aunque se consideró también a otros componentes de los contextos culturales. Uno de los puntos sobresalientes fue el desarmado de denominaciones 
tradicionales amplias que incluían materiales tempranos, como cultura de Los Barreales o cultura draconiana, las cuales englobaban lo que, desde su perspectiva, eran culturas diferentes. Luego, la elaboración de la secuencia maestra del valle de Hualfín a partir del cruce entre piezas de la colección Muñiz Barreto con material obtenido en capa y fechados radiocarbónicos, proporcionó un marco de referencia más ajustado para la investigación arqueológica del área valliserrana (e.g. González 1963; González y Cowgill 1975); para los períodos Temprano y Medio, se proponía la secuencia cultural Condorhuasi-Ciénaga Aguada. La misma secuencia fue extendida por González para los valles de Yocavil y Calchaquí.

A comienzos de los años '60 se realizó la Tercera Expedición Arqueológica de la Universidad Nacional del Litoral (UNL), dirigida por E. Cigliano; uno de sus objetivos fue arrojar luz sobre la problemática cronológica del poblamiento del sur de Yocavil. Por primera vez se tomaban en cuenta conjuntamente distintos sitios del Valle en un enfoque regional, y se empleaba una metodología que incluía prospección con apoyo de aerofotografías, la selección de lugares adecuados y la práctica de campo; la búsqueda de material funerario ya no era la prioridad, sino que se privilegiaba la investigación de unidades residenciales (Tarragó 2003). A partir de los resultados de los trabajos de campo, se elaboró una propuesta cronológica basada en la aplicación de seriación cuantitativa en el análisis de la cerámica (es decir, frecuencias de fragmentos según sus tendencias cuantitativas en la estratigrafía); para momentos tempranos, se planteaba el asentamiento en el Valle de la cultura Ciénaga, y se manifestaban dudas respecto a la existencia de una facie Aguada, dada la escasa presencia de fragmentos cerámicos de este estilo (Cigliano et al. 1960). Aunque esta secuencia se apoyaba en parte en la de Hualfín, se señalaba la presencia de una cerámica gris-negro pulida, de identidad propia, previa a Ciénaga y distinta de Candelaria, la cual según Cigliano sería la alfarería diagnóstica más temprana en el sur del valle de Yocavil (Cigliano 1959-1960; Cigliano et al. 1960).

La Quinta Expedición Arqueológica, dirigida por P. Krapovickas, puso atención en la zona de Tolombón, en el norte de Yocavil. En ese marco se excavaron áreas de vivienda de momentos previos a los Desarrollos Regionales, hallándose pisos de ocupación asociados a cerámica Candelaria (Tarragó 2003). Posteriormente se efectuaron otras incursiones: las excavaciones de Carrara y colaboradoras en los alrededores de Cafayate - sobre todo en el sitio formativo Chimpa - (Tarragó y Scattolin 1999) y los trabajos de campo en la zona de San Carlos comandados por Heredia, a partir de los cuales se definieron fases cronológicas para el sur del valle Calchaquí en su punto de unión con Yocavil (Heredia et al. 1974).

El estudio sistemático del área vallista fue interrumpido con el golpe de estado de 1966, el cual truncó el proceso académico gestado en la UNL (Tarragó 2003).

\section{En busca del tiempo perdido (Tercera y Cuarta Etapa)}

Los trabajos de campo en el sur de los valles Calchaquíes estuvieron interrumpidos hasta entrados los años '70, momento en el cual el objetivo de las investigaciones se centró en las poblaciones tardías (e. g. Podestá y Perrota 1973).

La desarticulación del creativo clima académico de la primera mitad de los '70, consecuencia del golpe militar del año '76, afectó especialmente a la producción arqueológica del NOA, terreno fértil para propuestas materialistas consideradas subversivas por la 
dictadura. A mediados de los `80, ya en democracia, las investigaciones en el Valle fueron retomadas, principalmente por parte de $\mathrm{M}$. Tarragó y equipo, en coincidencia temporal con el inicio de la tercera etapa de los estudios ceramológicos del NOA. Los trabajos de campo y las producciones escritas se orientaron a la arqueología de momentos posteriores al $900 \mathrm{AD}$; hasta la fecha, se ha logrado una caracterización profunda de diversos aspectos de las sociedades tardías, siendo la cerámica objeto de numerosos trabajos (e. g. Nastri 2008; Palamarczuk 2008).

En términos de coincidencia temporal, la tercera etapa no posee exponentes en lo que se refiere a la alfarería temprana, con excepción del trabajo de Tarragó et al. (1988), en el cual se analizó a nivel tecnológico, formal y decorativo, cerámica de superficie recolectada en el poblado arqueológico de la Loma Rica de Shiquimil, incluyendo fragmentos considerados de momentos previos a los Desarrollos Regionales; los autores aislaron estándares de pasta para conformar grupos cerámicos; la distribución de estos grupos según los recintos fue considerada una variable cronológica de la ocupación de los distintos sectores del poblado. Se trata del primer trabajo en aplicar esta metodología en el Valle. Por otra parte, existe referencia a materiales tempranos del área cercana a Quilmes en el trabajo de Pellisero y Difrieri (1981), aunque sin un estudio puntual de los mismos.

La investigación de la cerámica de las sociedades anteriores al 900 AD cobró impulso recién a partir los años '90 - en coincidencia cronológica con la cuarta etapa del modelo revisado - con las contribuciones de M. C. Scattolin y equipo en el centro y sur del Valle (e. g. Bugliani y Pereyra Domingorena 1999; Scattolin 2000, 2003a), y más adelante, del grupo de investigación de M. Tarragó en el sector meridional (e. g. Baigorria Di Scala y Spano 2007; Palamarczuk et al. 2007; Álvarez Larrain et al. 2009), y de M. Lo Celso y R. Ledesma en el sector septentrional$^{2}$ (e. g. Lo Celso y Ledesma 2004, 2005; Ledesma 2006-2007). También se contó con la primera síntesis acerca del estado de conocimiento sobre el Formativo local, con numerosas alusiones a la alfarería (Tarragó y Scattolin 1999). Otras producciones apuntaron el hallazgo de cerámica temprana en Pichao - centro del Valle - (Núñez Regueiro y Tartusi 1993) y en sitios de la quebrada de Tolombón - en el norte - (Williams 2003).

Los estudios contextuales han cumplido un rol clave en la búsqueda de un conocimiento mayor sobre las sociedades aldeanas del Valle. El primer exponente de esta tendencia fue la revisión de la colección Schreiter depositada en Gotenburgo (Stenborg y Muñoz 1999); si bien en la colección también hay piezas tardías, los materiales tempranos fueron protagonistas tanto por el número de fotografías presentadas como por los análisis de los cuales fueron objeto, que incluyeron los primeros fechados de momentos pre-tardíos en el Valle. En la línea de estudios anteriores respecto a las particularidades del Formativo en Yocavil (Tarragó y Scattolin 1999; Scattolin 2000), Scattolin (2003a) informó sobre piezas tempranas del norte del Valle hasta entonces desconocidas, depositadas en el Field Museum de Chicago, dando cuenta de los recursos estilísticos plasmados en las mismas. El interés de la autora por aspectos iconográficos de los objetos presantamarianos de colecciones se manifestó también en trabajos posteriores: el estudio de ceramios de la colección Muñiz Barreto procedentes de Laguna Blanca (Scattolin y Bugliani 2005) y el análisis del repertorio iconográfico de la cerámica temprana del Valle y áreas aledañas a partir de la revisión de materiales de excavación y de colecciones, con especial acento en la discusión del carácter local del fenómeno Aguada (Scattolin 2006b). 
En paralelo con el interés por la cerámica temprana, se dio la profusión de análisis de corte simbólico. Los estudios de colecciones implicaron un aumento del corpus de datos disponible, y nuevas preguntas y temáticas fueron desarrolladas. En sus trabajos de 2003b y 2005, Scattolin se interesó por la representación de la mujer en la iconografía temprana vallista (incluyendo a la cerámica entre otros soportes) desde una perspectiva de género, discutiendo asimismo la construcción del conocimiento sobre las figuras sexuadas en la arqueología del NOA. Esta puesta en conflicto de conceptos usados habitualmente en los estudios arqueológicos aparece también en otros trabajos de la autora (2004 y 2006a), los cuales discuten las categorías empleadas en las clasificaciones propuestas para el NOA, analizando los esquemas conceptuales a los que remiten dichas clasificaciones; el caso de estudio una vez más es el valle de Yocavil durante el primer milenio de la Era. Por su parte, Bugliani (2004) indagó acerca de las representaciones de la figura humana, apoyándose en el estudio de piezas de colecciones, proponiendo una aproximación a los sistemas de representaciones de las sociedades tempranas; en otro trabajo, la autora examinó las imágenes plasmadas en la alfarería funeraria temprana del sur de los valles Calchaquíes, mediante el análisis iconográfico, formal y contextual de piezas de la colección Muñiz Barreto, con el objetivo de lograr un acercamiento a los recursos plásticos empleados y a los modos de representación (Bugliani 2007). Estas temáticas fueron incluidas a su vez en un estudio mayor, en el cual la cerámica procedente de diversos sitios y también de colecciones fue analizada desde la perspectiva de la arqueología del consumo (Bugliani 2008). Asimismo, en un análisis de los recursos estilísticos de alfarería temprana asociada a contextos domésticos y funerarios del sitio Soria 2, se ha propuesto un uso simbólico de las técnicas decorativas, además del papel cumplido al respecto por la iconografía (Spano 2008).

La cerámica ordinaria ha sido considerada en varios trabajos con distinto énfasis según los objetivos perseguidos. Se la incluyó en el análisis del repertorio cerámico general en varios trabajos, como Bugliani y Pereyra Domingorena 1999, Fraga 1999, Baigorria Di Scala y Spano 2007, Bugliani 2008, y Álvarez Larrain et al. 2009. Es pertinente destacar algunas de estas contribuciones. El análisis de Bugliani y Pereyra Domingorena (1999) a partir de fragmentos del sitio Bañado Viejo, incluyó la descripción de estándares de pasta y de las decoraciones, y el cruce de ambos aspectos en la conformación de conjuntos analíticos. Fraga (1999) efectuó un estudio funcional de la alfarería de Morro de las Espinillas, privilegiando el aspecto morfológico de las piezas reconstruidas. Por otro lado, la alfarería ordinaria del sitio Soria 2 fue objeto de estudio de dos trabajos específicos: en Álvarez Larrain et al. 2006 se describieron las características estilísticas de ollas ordinarias empleadas en el entierro de niños, y en Baigorria Di Scala 2009 se analizó gran parte del conjunto ordinario de uso cotidiano, desde una perspectiva tecno-morfológica.

\section{Ayer y hoy: reflexiones sobre desfasajes y puestas al día}

A lo largo de esta revisión, se observa cómo pueden identificarse sucesivamente en los estudios de la alfarería temprana de Yocavil, las características distintivas de cada una de las etapas propuestas por Ramundo (2008). Es decir, podemos plantear que en un sentido general, la hipótesis de partida de nuestra indagación es en principio correcta, ya que la investigación en el Valle acompañó las tendencias teórico-metodológicas vigentes a lo largo de las décadas. Pero al mismo tiempo, el examen bibliográfico reveló que existen particularidades que se desvían del modelo general del NOA: ciertos interrogantes fueron 
planteados años después que en otras áreas, de modo que se halla en curso la búsqueda de las respuestas correspondientes.

Las particularidades se relacionan con el devenir de la arqueología del área, básicamente con el lugar relegado que tuvieron las poblaciones tempranas en la construcción arqueológica del pasado vallisto. Interrumpido el proyecto de investigación regional encabezado en su momento por Cigliano y luego por Krapovickas, el Formativo permaneció durante décadas en sombras (Tarragó y Scattolin 1999), a diferencia del estado de conocimiento de ese período en otras áreas, y de otros períodos en el mismo sector geográfico. Palamarczuk (2005) ha sostenido que en los últimos cuarenta años de investigación del ámbito de Yocavil estuvieron dadas las condiciones para profundizar las investigaciones sobre las sociedades agropastoriles tempranas, tomando como base los trabajos de Cigliano y equipo en diversos sitios (Cigliano et al. 1960); es decir, las propuestas cronológicas y las descripciones de la cerámica hallada constituían una base promisoria para profundizar la temática del Formativo en Yocavil. Sin embargo, la necesidad de identificación de culturas arqueológicas derivó en un oscurecimiento de las particularidades locales en pos de lograr una síntesis para toda la región. Otro factor a tener en cuenta estaría dado por los objetivos propios de los proyectos de investigación de los equipos que desde mediados de los ${ }^{\prime} 80$ han estado trabajando en el área, los cuales se orientaron al estudio de los grandes centros poblados tardíos y sus vinculaciones con los incas y los europeos (Palamarczuk 2005). Por lo tanto, es recién a partir de la conformación de un equipo específicamente interesado en el Formativo (dirigido por Scattolin) que se inicia la búsqueda de sitios tempranos con las consiguientes excavaciones de los mismos, la revisión de colecciones y la discusión en general respecto a la problemática de las sociedades aldeanas. A partir del año 2000 se sumaron los aportes de Tarragó y colaboradores en el sur del valle, y de Lo Celso y Ledesma en el norte. De este modo, en la actualidad el abordaje de la cerámica temprana de Yocavil es un potencial terreno fértil de discusión que involucra distintos aspectos de la investigación ceramológica y diferentes problemáticas del estudio de las sociedades pasadas: la cronología, el simbolismo, la producción, el uso o consumo de objetos, la definición estilística, la adscripción étnica, las interacciones entre poblaciones a distintas escalas, entre otros (en la Figura 2 se reproducen algunos exponentes de la cerámica temprana de Yocavil, referidos en producciones de los últimos años). Veamos de qué modo ciertos intereses característicos de etapas previas de la investigación ceramológica, constituyen puntos centrales en las producciones académicas de años recientes, confluyendo así en el estado actual de la cuestión.

El interés por la cerámica como índice cronológico (característico de la segunda etapa) se materializó en varios trabajos de Scattolin (2000; 2004; 2006a; 2006b; Scattolin et al. 2001), en los cuales las preocupaciones recurrentes expresadas son la falta de una secuencia local y la crítica a extrapolaciones de la secuencia maestra de Hualfín. Recientemente la autora ha propuesto tres fases dentro del primer milenio AD (Scattolin 2007a) ${ }^{3}$, a partir del cruce entre la variación diacrónica de los materiales de la columna estratigráfica del sitio Bañado Viejo (Scattolin et al. 2001), y el estudio de cerámica en posición primaria asociada a fechados seguros y de piezas de colecciones, conjuntos de superficie y fragmentos excavados sin datación absoluta asociada. Se incluyó en el espacio bajo estudio al valle y sierra del Cajón, la zona de la Candelaria, el área pedemontana de Tafí y la falda occidental del Aconquija. Se propuso entonces una ordenación temporal de los atributos cerámicos, la cual no tiene carácter definitivo, según expresa la autora, sino que plantea una integración del estado de conocimiento actual de la alfarería temprana. Los pasos seguidos de alguna manera siguen 


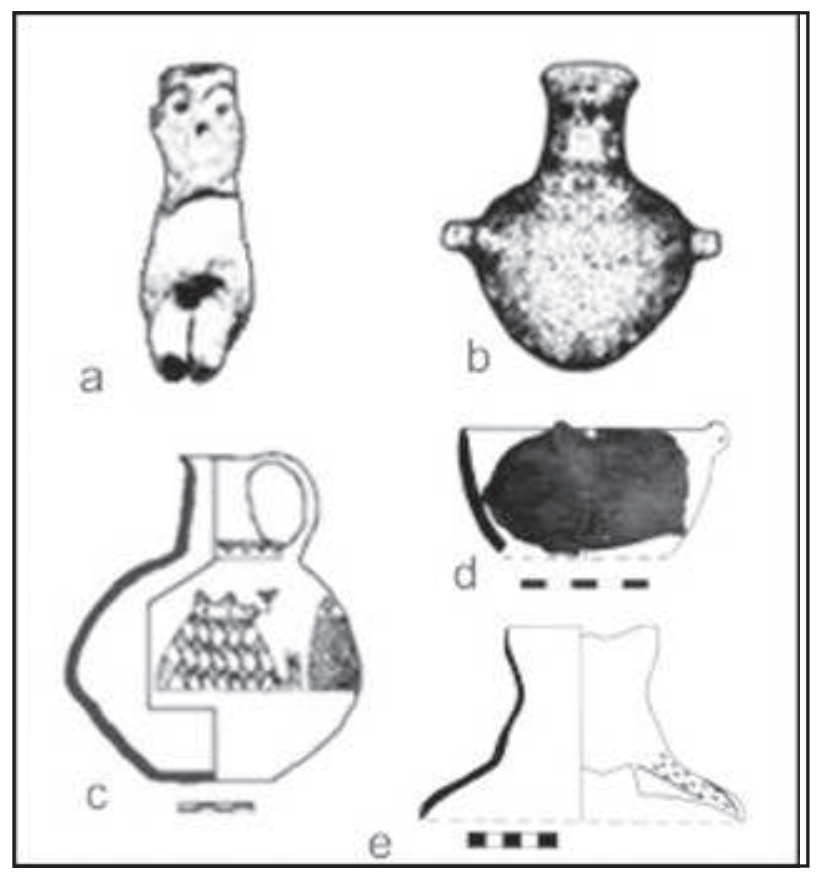

Figura 2. Exponentes de cerámica temprana de Yocavil; $a$ : pieza procedente de Cafayate (Scattolin 2006b); b: pieza recuperada en una tumba de Lampacito (Bugliani 2004); c: vasija hallada en La Banda de Arriba, Cafayate, asociada a un contexto funerario (Ledesma 20062007); d y e: cerámica de contexto doméstico recuperada en Soria 2 (d: Spano 2008, e: Alvarez Larrain et al. 2009).

la trayectoria metodológica empleada por ejemplo en Hualfín, Catamarca (González 1963) o en el área de La Candelaria, Salta (Heredia 1974).

En relación a la temática de la cronología, un elemento incorporado en tiempos relativamente recientes es la búsqueda y estudio de sitios habitacionales - característica que también se había sumado como interés naciente a los estudios ceramológicos en la segunda etapa (González 1955; Tarragó 2003), de modo de contar con información contextual acerca de las vasijas de momentos tempranos y complementar así los datos aportados por los estudios de colecciones, cuyas piezas proceden de contextos no cotidianos (funerarios, rituales, etc.). Muestra de ello son las investigaciones llevadas a cabo en sitios emplazados propiamente en el Valle, como Morro de las Espinillas (Fraga 1999; Scattolin 2007b) y Soria 2 (Spengler y Pérez 2006; Álvarez Larrain et al. 2009; Palamarczuk et al. 2007; Spano 2008), o en espacios adyacentes, como el valle y sierra del Cajón, y el valle de Tafí (Scattolin y Gero 1999; Nastri et al. 2004; Pastor y Rivero 2004; Scattolin et al. 2006, 2007).

Existen estudios que remiten a las características de la tercera etapa. Por ejemplo, la cerámica ha sido considerada un indicador arqueológico más, conjuntamente con otras líneas de evidencia material, como una vía para establecer las características de las ocupaciones formativas en la zona de Cafayate (Ledesma y Subelza 2009). También, las autoras establecieron grupos cerámicos de referencia para la localidad arqueológica La Banda de Arriba, en base a variables cualitativas, y refirieron a estudios de determinación de composición físico-química de fuentes de arcilla locales (Subelza 2008, citado en Ledesma y Subelza 2009). 
La atención a la variable tecnológica se manifestó en el estudio de la cerámica de Bañado Viejo (Bugliani y Pereyra Domingorena 1999), en cuya metodología la composición de las pastas fue un factor relevante en la conformación de conjuntos analíticos. El análisis petrográfico fue central en el estudio de vasijas del vecino valle del Cajón, como modo de aproximación a la interacción entre poblaciones tempranas (Pereyra Domingorena 2009); también fue un recurso analítico en estudios estilísticos y tecno-morfológicos de cerámica del sitio Soria 2 (Spano 2008; Baigorria Di Scala 2009).

La importancia de las tareas de remontaje para la reconstrucción de formas de vasijas ordinarias y la evaluación de procesos posdepositacionales, es el eje del trabajo de Álvarez Larrain et al. 2006, referido también a materiales de Soria 2. Asimismo, se han efectuado estudios químicos de residuos, para la determinación de las sustancias consumidas en pipas cerámicas del Formativo vallisto (Rosso y Spano 2005-2006; Scattolin et al. 2009).

Los estudios estadísticos están representados por el trabajo de Bugliani (2009), en el cual se sistematizó la variabilidad morfológica de piezas de los cementerios tempranos de la falda occidental del Aconquija, mediante el empleo de análisis multivariado.

Si a estos "desfasajes" en cuanto al modelo general de investigaciones en el NOA, le sumamos las referidas perspectivas actuales que se encuentran en sincronía con la cuarta etapa, se conforma un panorama sumamente variado en cuanto a visiones respecto a la cerámica como objeto de estudio. Reflexionando sobre esta situación, podemos plantear tres cuestiones. La primera es que la multiplicidad de preguntas que se encontraban pendientes, de alguna manera propició un panorama activo de indagaciones respecto a distintas problemáticas, en el cual los elementos característicos de las sucesivas etapas de los estudios ceramológicos en el NOA, en Yocavil resultan ser una suerte de "casilleros" que se van llenando de manera más o menos simultánea: un casillero destinado a la cronología, otro a los procesos sociales a distinta escala, otro al simbolismo, etc., construyéndose de este modo versiones del relato arqueológico acerca de las sociedades aldeanas que empiezan a contrarrestar la aparente invisibilidad arqueológica que tuvo el Formativo local.

La segunda cuestión alude a uno de los ejes de la producción académica actual referida a la cerámica temprana en el área. Si bien durante mucho tiempo las referencias a las sociedades tempranas de Yocavil estuvieron teñidas por alusiones a la secuencia maestra de Hualfín, es atendible considerar el impacto que la misma tuvo en la historia de la arqueología del NOA en general, en un momento en el cual la ausencia de definiciones cronológicas certeras era la principal deuda de la disciplina en nuestro país (Núñez Regueiro 1961-1964); ante una visión más bien plana de la trayectoria de las poblaciones prehispánicas, las propuestas generadas con mayor refinamiento metodológico por A. R. González implicaron progresivamente una reorganización conceptual del pasado, tanto a partir de la sucesión cronológica como de la identificación de culturas, plasmadas en la periodificación del noroeste. El área de Yocavil no quedó exenta de esta tendencia, pero en lugar de contarse con una secuencia propia, se planteó una correlación entre los sucesivos procesos sociales locales, y aquellos sostenidos para los valles meridionales (González 1963); esta propuesta fue un punto de referencia tenido en cuenta durante mucho tiempo, ante la ausencia de un modelo cronológico-cultural local. Es necesario aclarar al respecto que el mismo González advirtió en varios de sus trabajos que la secuencia debía estar siempre sujeta a ajustes y revisiones 
(González 1955: 14; González y Cowgill 1975: 387), de modo que nunca la consideró una estructura cerrada.

En tercer lugar, no se ha encontrado hasta el momento correspondencia estilística entre la cerámica temprana del Valle y otros soportes materiales (como el arte rupestre, la metalurgia, la lapidaria, etc.). Claramente, en otras áreas y en otros períodos, la enunciación de un estilo para designar una producción artefactual amplia ha otorgado identidad arqueológica a las poblaciones bajo estudio (e. g. González 1998 para el caso Aguada; Tarragó et al. 1997 para el estilo santamariano). Además, en el análisis efectuado hasta la fecha de la cerámica temprana originaria de Yocavil, no se ha contado con la seguridad que confiere el uso de términos como Ciénaga o Condorhuasi, entre otros, en el sentido de "sitios tipos" y de las consiguientes denominaciones estilísticas o tipológicas para la alfarería, y este hecho se relaciona obviamente con los avatares de la historia de la investigación arqueológica: la manera de construir el conocimiento se vincula necesariamente con la elaboración de categorías operativas (Spano 2008).

\section{Conclusiones}

Consideramos que el modelo historiográfico de Ramundo (2008) puede ser empleado en el caso particular de la cerámica temprana de Yocavil, en tanto sea entendido como una de las sistematizaciones posibles de la información disponible sobre el tema; pero esta aplicación no debe ser rígida: si bien existe una tendencia diacrónica que se corresponde con el modelo de cuatro etapas, los rasgos propios de la investigación arqueológica en Yocavil exigen que el modelo sea tomado como una referencia flexible, sobre todo en lo que respecta a las producciones académicas de los últimos diez años.

Reflexionando sobre las cuestiones aludidas en este artículo, podemos retomar a modo de cierre planteos efectuados hace tiempo por Núñez Regueiro (1975) en relación al proceso operado en los distintos ensayos de periodificación en el NOA; el autor propone tres niveles necesarios en el camino hacia la obtención de cualquier periodificación. En un primer nivel, se distribuyen los materiales arqueológicos en el tiempo, previamente a abordar el desarrollo histórico de una determinada región; se buscan regularidades en los materiales bajo estudio, básicamente en la cerámica, pero los fundamentos para la periodificación son aún limitados. En un segundo nivel, se emplean criterios más adecuados, en virtud de que se produzcan avances en el conocimiento y mayor disponibilidad de marcos teóricos para abordar la cuestión; estos criterios aluden a la consideración sucesiva de (a) otras líneas de evidencia además de la cerámica, sumado al estudio de aspectos como las costumbres funerarias y la vivienda, entre otros; y (b) la organización sociopolítica y económica, y la incidencia de factores ambientales; al mismo tiempo se da un ajuste en la cronología. Finalmente existe una tercera instancia, en la cual la periodificación debe reflejar los niveles de desarrollo de las entidades socioculturales del pasado, manifestados por estructuras particulares; dichos niveles son resultado del progreso en los modos de producción. Al momento de escribir ese artículo, el autor consideraba que en las propuestas regionales las distintas subáreas se encontraban en niveles dispares, en función del respectivo estado de conocimiento. A nuestro criterio, la construcción del relato arqueológico del pasado profundo en el valle de Yocavil se encuentra en el segundo nivel propuesto por Núñez Regueiro, debido a la ampliación del conocimiento sobre los distintos aspectos de las sociedades aldeanas que se viene produciendo en los últimos años, sumado al crecimiento de la base de datos sobre fechados radiocarbónicos 
(para una síntesis ver Scattolin 2007a); existen aún muchas puertas que esperan por ser abiertas, de modo que el tercer nivel aún se encuentra lejos de alcance. La cerámica parece haber sido el disparador clave en el sinuoso camino iniciado hacia la comprensión del pasado aldeano de Yocavil; los pasos futuros permitirán avanzar en esta travesía que, claro está, nunca habrá de completarse del todo.

\section{Agradecimientos}

A Paola Ramundo y Javier Nastri, por sus comentarios y sugerencias sobre la versión original de este trabajo, y a Juan Manuel Estevez y Sol Grimodi, por su colaboración técnica. Agradezco a los evaluadores anónimos, cuyas observaciones mejoraron la versión final. De todos modos, lo expresado es de mi exclusiva responsabilidad.

\section{Notas}

1- En el artículo se emplean los términos Temprano y Formativo indistintamente, y aplicados a la dimensión cronológica de la definición de dichos vocablos.

2- En las referencias citadas las autoras han incluido a la micro-región de Cafayate - en la cual se emplazan los sitios estudiados por ellas - dentro del área del Valle Calchaquí sur (Lo Celso y Ledesma 2005; Ledesma y Subelza 2009), y consideraron al Valle de Santa María o Yocavil como un área adyacente (Lo Celso y Ledesma 2005: 267).

3- En la investigación ceramológica de Yocavil, a esta secuencia se sumó la propuesta de fases cerámicas para la localidad arqueológica de Rincón Chico (Greco 2007), aunque tal estudio cronológico corresponde temporalmente a los Desarrollos Regionales.

\section{Bibliografía}

Álvarez Larrain, A., J. Baigorria Di Scala, C. Belotti, J. P. Carbonelli, E. Figueira, S. Grimoldi, M. S. López, D. Magnifico, V. Palamarczuk, J. Ponce de León, R. Spano, L. Stern Gelman y F. Weber

2006. Tres ollas cerámicas empleadas en el entierro de párvulos en un espacio doméstico formativo en el valle de Yocavil, provincia de Catamarca, Argentina. Presentado en las VII Jornadas de Jóvenes Investigadores en Ciencias Antropológicas, INAPL, Bs. As. Ms.

Álvarez Larrain, A., J. Baigorria Di Scala, C. Belotti, J. P. Carbonelli, S. Grimoldi, M. S. López; D. Magnifico, V. Palamarczuk, J. Ponce de León, R. Spano, G. Spengler, L. Stern Gelman y F. Weber

2009. Avances en el estudio de un contexto doméstico formativo en el Valle de Yocavil. Entre pasados y presentes II, Estudios Contemporáneos en Ciencias Antropológicas (ed. por T. Bourlot, D. Bozzuto, C. Crespo, A. C. Hecht, N. Kuperszmit), pp. 369-382. Fundación de Historia Natural Félix de Azara - INAPL, Bs. As.

Ambrosetti, J. B.

1892. Descripción de algunas alfarerías calchaquíes depositadas en el Museo Provincial de Entre Ríos. Revista del Museo de La Plata 3: 67-79.

1896. El símbolo de la serpiente en la alfarería funeraria de la región calchaquí. Boletín del Instituto Geográfico Argentino 17: 219-230.

Baigorria Di Scala, L. J.

2009. El sitio Formativo Soria 2: Estudio Tecno Morfológico del conjunto cerámico ordinario. Tesis de Licenciatura en Cs. Antropológicas (orientación Arqueología). F. F. y L., UBA. No publicada. 
Baigorria Di Scala y R. C. Spano

2007. Análisis preliminar de la cerámica del sitio formativo Soria 2 (Valle de Yocavil, Catamarca). Actas del XVI Congreso Nacional de Arqueología Argentina, Número Especial Revista Pacarina tomo III, pp. 65-70. EdiUnju, San Salvador de Jujuy.

Balesta, B. M. y V. I. Williams

2007. El análisis cerámico desde 1936 hasta nuestros días. Relaciones de la Sociedad Argentina de Antropología 32: 169-190.

Bengtsson, L., P. Cornell, N. Johansson y S. Sjödin

2001. Investigations at Pichao. Introduction to studies in the Santa Maria Valley, Northwestern Argentina. BAR 978, Oxford.

Bennet, W. C., E. F. Bleiler y F. H. Sommer

1948. Northwest Argentine Archaeology. Yale University Publications in Anthropology 38.

Bugliani, M. F.

2004. Formas y recursos estilísticos para la representación humana durante el Formativo en el Valle de Santa María. Acta Americana 12 (1): 79-88.

2007. Representaciones visuales y prácticas en contextos funerarios. Las vasijas del Formativo en las tumbas del sur de los Valles Calchaquíes. Actas del XVI Congreso Nacional de Arqueología Argentina, Número Especial Revista Pacarina, tomo II, pp. 421-426. EdiUnJu, San Salvador de Jujuy.

2008. Consumo y representación en el Formativo del sur de los Valles Calchaquíes (Noroeste argentino): Los conjuntos cerámicos de las aldeas del primer milenio A.D. BAR, International Series, S1174. Oxford.

2009. Métodos de ordenación y técnicas de agrupamiento aplicadas a los recipientes cerámicos: las vasijas de los cementerios de la falda occidental del Aconquija. Arqueometría latinoamericana, $2^{\circ}$ Congreso Argentino, $1^{\circ}$ Latinoamericano (ed. por O. Palacios, C. Vázquez, T. Palacios y E. Cabanillas), vol. 2, pp. 345-350. CNEA. Bs. As.

Bugliani, M. F. y L. Pereyra Domingorena

1999. Conjuntos cerámicos en el sitio formativo de "Bañado Viejo" (Tucumán). Actas del XIII Congreso Nacional de Arqueología Argentina, tomo II, pp. 347-358. Córdoba.

Cigliano, E. M.

1959-1960. Nuevos aportes sobre las primeras culturas alfarero-agrícolas del valle de Santa María. Acta Prehistórica 3-4: 150-152.

Cigliano, E. M., M. L. Arocena, B. Carnevali, M. T. Carrara, G. De Gásperi, A. M. Lorandi, S. Petruzzi, S. Renard y M. Tarragó

1960. Investigaciones Arqueológicas en el Valle de Santa María. Publicación 4. Instituto de Antropología, Facultad de Filosofía y Letras, UNL, Rosario.

Debenedetti, S.

1912. Noticias sobre una urna antropomórfica del valle de Yocavil. Revista del Museo de La Plata 23: 196-205. 
Fernández, J.

1982. Historia de la Arqueología Argentina. Anales de Arqueología y Etnología 34/35 (320 páginas).

Fraga, C.

1999. Cerámica: un Estudio Morfológico Funcional en el Valle de Santa María. Tesis de Licenciatura en Ciencias Antropológicas (orientación Arqueología), F. F. y L., UBA. No publicada.

García, L. C. y M. F. Kusch

1994. Confluencia de visiones sobre la cerámica arqueológica desde la ciencia y la artesanía. Arqueología 4: 235-246.

González, A. R.

1955. Contextos y secuencias culturales en el Área Central del Noroeste Argentino (Nota preliminar). XXXI Congreso Internacional de Americanistas (Sao Paulo), pp. 699-725.

1960: Nuevas fechas de la cronología Arqueológica Argentina obtenidas por el método de Radiocarbón (IV). Resumen y Perspectivas. Revista del Instituto de Antropología 1:303-331.

1963. Cultural Development in NW Argentina. Aboriginal Development in Latin America: An Interpretative Review (ed. por B. Meggers y C. Evans), pp. 103-117. Smithsonian Miscellaneous Collection 1, Washington.

1998. Cultura La Aguada. Arqueología y diseños. Filmediciones Valero. Bs. As.

González, A. R. y G. L. Cowgill

1975. Cronología arqueológica del Valle de Hualfín, Pcia. de Catamarca, Argentina, obtenida mediante el uso de computadoras. Actas del I Congreso Nacional de Arqueología Argentina (Rosario, 1970), pp. 383-404. Bs. As

Greco, C.

2007. Secuencias radiocarbónicas y estilos cerámicos en Rincón Chico, Valle de Yocavil, Catamarca. Tesis de Licenciatura en Cs. Antropológicas (orientación Arqueología), F. F. y L., UBA. No publicada.

Heredia, O. R.

1974. Investigaciones arqueológicas en el sector meridional de las Selvas Occidentales. Revista del Instituto de Antropología 5: 73-132.

Heredia, O. R., M. Palacios, A. Luzzi y L. Naudeau

1974. Ensayo de un cuadro cronológico del sector meridional del Valle Calchaquí. Presentado en el III Congreso Nacional de Arqueología Argentina, Salta. Ms.

Ledesma, R.

2006-2007. Integración de sitios con arte rupestre y su territorio en la microrregión Cafayate (provincia de Salta). Cuadernos del INAPL 21: 115-131.

Ledesma, R. y C. Subelza

2009. Alcances y limitaciones para caracterizar las ocupaciones formativas en Cafayate (Salta). Andes 20. En prensa. 
Liberani, I. y R. Hernández

1956 [1877]. Excursión arqueológica en los valles de Santa María, Catamarca, 1877. Instituto de Antropología, UNT., S. M. de Tucumán.

Lo Celso, M. G. y R. E. Ledesma

2004. Aportes sobre evidencias Formativas en el municipio de Cafayate (Salta). Libro de resúmenes del XV Congreso Nacional de Arqueología Argentina, Río Cuarto: pp. 313. Universidad Nacional de Río Cuarto.

2005. Sitios arqueológicos formativos en el Municipio de Cafayate (Salta). Avances de Investigación. I Jornadas de Antropología, Facultad de Humanidades, Universidad Nacional de Salta: 267- 282.

Madrazo, G.

1985. Determinantes y orientaciones en la antropología argentina. Boletín del Instituto Interdisciplinario de Tilcara 1: 13-56.

Nastri, J. H.

2004. Los primeros americanistas (1876-1900) y la construcción arqueológica del pasado en los Valles Calchaquíes (Noroeste Argentino). Hacia una arqueología de las arqueologías sudamericanas (ed. por A. Haber), pp. 91-114. Universidad de Los Andes, Bogotá.

2008. La figura de las largas cejas de la iconografía santamariana. Chamanismo, sacrificio y cosmovisión calchaquí. Boletín del Museo Chileno de Arte Precolombino 13 (1): 9-34.

Nastri, J. H., G. Pratolongo, A. D. Reynoso y A. M. Vargas

2004. Arqueología de la Sierra del Cajón: poblados, corrales y pinturas. Ponencia presentada al XV Congreso Nacional de Arqueología Argentina. Universidad Nacional de Río Cuarto. En Prensa. Resumen publicado disponible en el Libro de resúmenes y programa general de actividades: pp. 317.

Núñez Regueiro, V. A.

1961-1964.Posibilidades y necesidad de aplicación de un método cuantitativo para obtener cronología cultural. Revista del Instituto de Arqueología 2/3: 197-202.

1975. El problema de la periodificación en arqueología. Etnia 16 (Suplemento Actualidad Antropológica): 1-20.

Núñez Regueiro, V. A. y M. R. A. Tartusi

1993. Orígenes de la Ocupación Prehispánica del Sitio StucTav 5 (El Pichao), Provincia de Tucumán. Publicaciones del Inst. de Arqueología de Tucumán 2: 19-30.

Palamarczuk, V.

2005. Breve ensayo sobre las representaciones temporales y culturales relativas a la arqueología del Valle de Santa María. Ms.

2008. Un análisis de la cerámica arqueológica de cuatro sitios en el bajo de Rincón Chico. Estudios Arqueológicos en Yocavil (ed. por M. N. Tarragó y L. R. González), pp. 19-80. Asociación Amigos del Museo Etnográfico, Bs. As.

Palamarczuk, V., R. Spano, F. Weber, D. Magnifico, S. López y M. Manasiewicz 2007. Soria 2. Apuntes sobre un Sitio Formativo en el Valle de Yocavil (Catamarca, Argentina). Intersecciones en Antropología 8: 121-134. 
Pastor, S. y D. E. Rivero

2004. Nuevas evidencias en torno a la ocupación agroalfarera temprana del valle de Yocavil. Mosaico. Trabajos en Antropología Social y Arqueología, pp. 189-199. INAPL, Bs. As.

Pellisero, N. y H. A. Difrieri

1981. Quilmes. Gobierno de la Provincia de Tucumán, S. M. de Tucumán.

Pereyra Domingorena, L.

2009. Análisis petrográfico de los recipientes del sitio Cardonal. Arqueometría latinoamericana, $2^{\circ}$ Congreso Argentino, $1^{\circ}$ Latinoamericano (ed. por O. Palacios, C. Vázquez, T. Palacios y E. Cabanillas), vol. 1, pp. 40-46. CNEA. Bs. As.

Podestá, C. y E. B. Perrota

1973. Relaciones entre culturas del noroeste argentino. San José y Santa María. Antiquitas 17: 6-15.

Ramundo, P.S.

2008. Estudio historiográfico de las investigaciones sobre cerámica arqueológica en el Noroeste Argentino. BAR Internacional Series. Archaeopress, Oxford.

Rosso, C. y R. C. Spano

2005-2006. Evidencias del uso de alucinógenos en pipas halladas en dos sitios tempranos de los Valles Calchaquíes. Arqueología 13: 79-98.

Scattolin, M. C.

2000. Santa María durante el Primer Milenio A. D. ¿Tierra Baldía? Årstryck 1995-1998: 63-83. Etnografiska Museet i Göteborg.

2003a. Los ancestros de Calchaquí: una visión de la colección Zavaleta. Cuadernos de la Facultad de Humanidades y Ciencias Sociales 20: 51-79.

2003b. Representaciones sexuadas y jerarquías sociales en el Noroeste Argentino Prehispánico. Acta Americana 11 (1): 30-48.

2004. Categorías indígenas y clasificaciones arqueológicas en el Noroeste Argentino. Hacia una arqueología de las arqueologías sudamericanas (ed. por A. Haber), pp. 53-82. Universidad de Los Andes, Bogotá.

2005. La mujer que carga el cántaro. Género y etnicidad en la arqueología sudamericana (ed. por V. Williams y B. Alberti), pp. 43-71. Serie Teórica 4. INCUAPA, UNICEN, Olavarría.

2006a. Categoremas indígenas y designaciones arqueológicas en el Noroeste Argentino prehispánico. Chungará 38 (2): 185-196.

2006b. Contornos y confines del universo iconográfico precalchaquí del valle de Santa María. Estudios Atacameños 32: 119-139.

2007a. Santa María antes del año mil. Fechas y materiales para una historia cultural. En Sociedades Precolombinas Surandinas. Temporalidad, Interacción y Dinámica cultural del NOA en el ámbito de los Andes Centro-Sur (ed. por V. I. Williams, B. N. Ventura, A. B. M. Callegari y H. D. Yacobaccio), pp. 203-219. Edición de los autores, Bs. As.

2007b. Estilos como recursos en el Noroeste Argentino. Procesos sociales prehispánicos en el sur andino, tomo I, La vivienda, la comunidad y el territorio (ed. por A. Nielsen, M. C. Rivolta, V. Seldes, M. Vázquez y P. Mercolli), pp. 291-321. Editorial Brujas, Córdoba. 
Scattolin, M. C. y M. F. Bugliani

2005. Un repertorio surtido: las vasijas de los oasis de Laguna Blanca, Puna Argentina. Revista Española de Antropología Americana 35: 51-74.

Scattolin, M. C., F. Bugliani, A. Izeta, M. Lázzari, L. Pereyra Domingorena y L. Martínez 2001. Conjuntos materiales en dimensión temporal. El sitio Formativo "Bañado Viejo" (Valle de Santa María, Tucumán). Relaciones de la Sociedad Argentina de Antropología 26: 167-192.

Scattolin, M. C., L. I. Cortés, M. F. Bugliani, C. M. Calo, L. Pereyra Domingorena, A. D. Izeta y M. Lazzari

2009. Built landscapes of everyday life: a house in an early agricultural village of northwestern Argentina. World Archaeology 41 (3): 396-414.

Scattolin, M. C., L. Cortés, L. Pereyra Domingorena, M. F. Bugliani, C. M. Calo, A. D. Izeta, M. Lázzari y J. Izaguirre

2007. Una aldea formativa en el Valle del Cajón. Actas del XVI Congreso Nacional de Arqueología Argentina, Número Especial Revista Pacarina tomo II, pp. 337-341. EdiUnJu, San Salvador de Jujuy.

Scattolin, M. C. y J. Gero

1999. Consideraciones sobre fechados radiocarbónicos en Yutopián, Catamarca, Argentina. Actas del XII Congreso Nacional de Arqueología Argentina, tomo I, pp. 65-73. La Plata.

Scattolin, M. C., L. Pereyra Domingorena, L. Cortés, M. F. Bugliani, C. M. Calo, A. D. Izeta y M. Lazzari

2006. Cardonal: una aldea formativa entre los territorios de valles y puna. Cuadernos de la Facultad de Humanidades y Ciencias Sociales 32: 211-225.

Serrano, A.

1958. Manual de cerámica indígena. Assandri, Córdoba.

Spano, R. C.

2008. Indagaciones sobre las sociedades aldeanas del Valle de Yocavil; análisis de la alfarería fina del sitio Soria 2 (Andalhuala, pcia. de Catamarca). Tesis de Licenciatura en Cs. Antropológicas (orientación en Arqueología). F. F. y L., UBA. No publicada.

Spengler, G. y M. Pérez

2006. Análisis preliminar de la distribución espacial y temporal del material de superficie del sitio Soria 2, Andalhuala (valle de Santa María, Catamarca). Ms.

Stenborg, P. y A. Muñoz

1999. Masked Histories. A Re-examination of the Rodolfo Schreiter Collection from North-western Argentina. Etnologiska Studier 43, Göteborg.

Tarragó, M. N.

1999. Las sociedades del sudeste andino. En Historia General de América Latina, v. 1: Las sociedades originarias, pp. 465-480. Trotta, Ediciones UNESCO. París.

2003. La arqueología de los Valles Calchaquíes en perspectiva histórica. Local, regional, global: Prehistoria, protohistoria e historia en los valles Calchaquies (ed. por P. Cornell y P. Stenborg), Anales Nueva Época 6: 13-42. Instituto Iberoamericano - Universidad de Göteborg, Göteborg. 
Tarragó, M. N., S. E. Caviglia, M. M. Peralta Sanhuesa y J. Sosa

1988. Los Grupos Cerámicos del Poblado de Loma Rica de Shiquimil, Catamarca Argentina. Trabajo presentado al IX Congreso Nacional de Arqueología Argentina, Bs. As. Ms.

Tarragó, M. N. y L. R. González

2008. Introducción: estudios arqueológicos en Yocavil. Estudios Arqueológicos en Yocavil (ed. por M. N. Tarragó y L. R. González), pp. 7-17. Asociación Amigos del Museo Etnográfico, Bs. As.

Tarragó, M. N., L. R. González y J. H. Nastri

1997. Las interacciones prehispánicas a través del estilo: el caso de la iconografía santamariana. Estudios Atacameños 14: 223-242.

Tarragó, M. N. y M. C. Scattolin

1999. La Problemática del Período Formativo en el Valle de Santa María. Actas del XII Congreso Nacional de Arqueología Argentina, tomo I, pp. 142-153. La Plata.

Williams, V.

2003. Nuevos datos sobre la prehistoria local en la quebrada de Tolombón. Pcia. de Salta. Argentina. Local, regional, global: Prehistoria, protohistoria e historia en los valles Calchaquies (ed. por P. Cornell y P. Stenborg), Anales Nueva Época 6: 165-210. Instituto Iberoamericano Universidad de Göteborg, Göteborg. 